\title{
Multifractal Analysis on the Sphere
}

\author{
Emilie Koenig and Pierre Chainais \\ LIMOS UMR 6158, University of Clermont-Ferrand II, 63170 Aubière Cedex, France \\ emilie.koenig@isima.fr, pchainai@isima.fr
}

\begin{abstract}
A new generation of instruments in astrophysics or vision now provide spherical data. These spherical data may present a selfsimilarity property while no spherical analysis tool is yet available to characterize this property. In this paper we present a first numerical study of the extension of multifractal analysis onto the sphere using spherical wavelet transforms. We use a model of multifractal spherical textures as a reference to test this approach. The results of the spherical analysis appear qualitatively satisfactory but not as accurate as those of the usual 2D multifractal analysis.
\end{abstract}

\section{Introduction}

Multifractal analysis is used to characterize the self-similarity property of objects. This analysis method has been used in various domains, each presenting self-similar data, such as turbulence in physics [1, network traffic [2, DNA series in biology [3] or in the study of natural images [4. The development of new instruments in several domains, as astrophysics [5] or vision [6], leads to a new generation of data: spherical data. These new elements may present a self-similarity property but no multifractal analysis has yet been proposed to characterize it. In this paper, we introduce a spherical multifractal analysis based on spherical wavelets as a natural extension of the usual 2D analysis. A numerical study is carried out with help of synthetic spherical textures generated by an extension of the Compound Poisson Cascade (CPC) model [7] on the sphere. These numerical experiments show that the spherical analysis appear qualitatively satisfactory but not as accurate as those of the usual 2D multifractal analysis.

The paper is organized as follows. First, we recall on the 2D multifractal analysis theory and introduce its extension onto the sphere. Next, we present a family of multifractal processes on the sphere, namely the Compound Poisson Cascades, to be used as a reference to test the method. Then, we perform numerical experiments to test the spherical multifractal analysis. Finally, we comment on our results and discuss about possible forthcoming improvements.

\section{Multifractal Analysis on the Sphere}

\section{$2.1 \quad 2 D$ Multifractal Analysis}

The self similarity property [8] refers to the fact that a part of an object resemble the whole object itself. This property can be associated to some deterministic 
geometrical feature: a dilated version of some part of a snowflake is similar to the entire snowflake. An object can also be self similar in a statistical sense. For example, a dilated segment of an Internet traffic signal is statistically similar to the whole signal. At least in some range no scale plays a specific role. Deterministic and stochastic fractals are examples of such objects. In the following, we will refer to an object for either a function or a realization of a stochastic process. Formally, the self similarity property is usually characterized by power law scalings, and more precisely by the evolution of partition functions $S_{q}(a)$ with scale $a$. For positive functions $f$, partition functions can be defined as estimates of the $q$ th-order moments of the localized box averages for a covering set of $N_{a}$ positions indexed by $l$ :

$$
S_{q}^{b o x}(a)=\frac{1}{N_{a}} \sum_{l=1}^{N_{a}} \varepsilon_{a}^{q}(l) \propto a^{\tau(q)} \text { where } \varepsilon_{a}(l)=\frac{1}{a} \int_{l-a / 2}^{l+a / 2} \beta_{0}\left(\frac{x-l}{a}\right) f(x) d x
$$

where $\beta_{0}$ is a positive weighting function. In the simplest case, $\beta_{0}=\mathbb{1}_{x \in[-1 / 2,1 / 2]}$. The coefficients $\varepsilon_{a}(l)$ are called aggregation coefficients 9]. For more general functions, the partition functions are often defined as wavelet-based partition functions as given by [2]:

$$
S_{q}^{s w t}\left(a=2^{j}\right)=\frac{1}{N_{j}} \sum_{l=1}^{N_{j}}\left|d_{f}(j, l)\right|^{q} \propto 2^{j \zeta(q)}
$$

where $d_{f}(j, l)$ are the $L^{1}$ normalized discrete wavelet coefficients of the object $f$ under study and $N_{j}$ is the number of wavelet coefficients at octave $j=\log _{2}(a)$. The function $\zeta(q)$ defines a set of so-called multifractal exponents. In the simplest case, $\zeta(q)=q H$ so that all the exponents are described by a single parameter $H$. The object is then called monofractal. A monofractal object is characterized by a unique fractal dimension. This is for instance the case of the fractional Brownian motion. In general, the function $\zeta(q)$ takes the form $\zeta(q)=q H+\tau(q)$ where the function $\tau(q)$ is a non-linear function such that $\tau(0)=\tau(1)=0$ and describes the deviation of $\zeta(q)$ from a linear function. The object is then called multifractal. The function $\zeta(q)$ is then characterized both by $H=\zeta(1)$ and $\tau(q)=\zeta(q)-q H$. The function $\tau(q)$ features the multifractality of the object. The estimated function $\zeta(q)$ may also be used as an input set of parameters for a model such as the Compound Poisson Cascades described in Sect. 3.1.

\subsection{Extension of the Multifractal Analysis to the Sphere}

We propose to extend the multifractal analysis described above to the sphere by using the same formulas but with spherical wavelets. We use a continuous spherical wavelet transform. The multifractal analysis is then only valid for $q \geq 0$ otherwise it will be numerically unstable and will raise theoretical problems [10. Different constructions of spherical wavelet transforms (SWT) have been proposed. 
P. Schröder and W. Sweldens [11] wanted to extend the discrete wavelet transform to any manifolds and in particular to the sphere. They have used a subdivision grid on the sphere (see Fig. 1(a) . Such a grid is based on the iterative division of the faces of an icosahedron in 4 new faces of equal area. These authors have constructed spherical wavelet functions with help of the lifting scheme and used these functions for compression. Two softwares are based on this transform: SD (http://www.multires.caltech.edu/software/sd/) developed by P. Schröder in 1998 and itkSWaveletSource [12] developed by Y. Gao et al. in 2007.

The extension of the wavelet construction made by J.-L Starck et al. [13] was motivated by the study of the cosmological microwave background (CMB) in astrophysics. These data are therefore mapped on the HEALPix (Hierarchical Equal Area isoLatitude Pixelization, http://healpix.jpl.nasa.gov) grid [14] (see Fig. 1(b) . The meshes of this grid are distributed on isolatitudes and have the same area so that every mesh has the same weight. The spherical wavelet construction of J.-L Starck et al. based on the spherical harmonic transform is implemented in a software available from the MRS Home Page (http://jstarck.free.fr/ mrs.html).

J.-P. Antoine et al. [15] have introduced a spherical wavelet transform based on a correspondence between the plane and the sphere. The translation and dilation used in the definition of the $2 \mathrm{D}$ wavelet transform are replaced respectively by the rotation and the stereographic dilation on the sphere. The rotation $R(\rho)$, where $\rho$ is a set of Euler angles, and dilation $D_{a}$ operators are then defined by

$$
\begin{gathered}
(R(\rho) f)(\omega)=f\left(R_{\rho}^{-1} \omega\right) . \\
\left(D_{a} f\right)(\omega)=\lambda(a, \theta)^{1 / 2} f\left(D_{a}^{-1} \omega\right) .
\end{gathered}
$$

where $f(\omega)$ is a function of the spherical coordinates $\omega=(\theta, \phi)$. The stereographic dilation maps the sphere without its South Pole on the plane. It imposes the use of an equiangular grid (see Fig. 1(c) . The two major defaults of this grid are the oversampling of the poles and the area variation of meshes. However, we chose this tool because it is efficient and easy to use in practice. The spherical continuous wavelet transform of $f(\omega)$ is the convolution between the function and the rotated and dilated versions of the mother wavelet $\Psi$

$$
W_{\Psi}^{f}(\rho, a)=\left\langle\Psi_{a, \rho} \mid f\right\rangle=\int_{S^{2}} d \mu(\omega) \overline{\left[R_{\rho} D_{a} \Psi\right](\omega)} f(\omega) .
$$

Then, the spherical multifractal analysis will be performed by replacing $d_{f}(j, l)$ by $W_{\Psi}^{f}(\rho, a)$ in (2) for a discrete sequence of tangential scales $a_{j}=$ $2 \tan \left((\pi / 4) 2^{-j}\right), 1 \leq j \leq J$. This wavelet transform is implemented in the Matlab toolbox YAWTb (Yet Another Wavelet Toolbox, developed by L. Jacques et al. in 2002, http://rhea.tele.ucl.ac.be/yawtb/) [16].

The multifractal coefficients $\zeta(q)$ can only be calculated using a $L^{1}$ normalized wavelet. In practice, if $\Psi^{(2)}$ is the $L^{2}$ normalized spherical wavelet function, the 


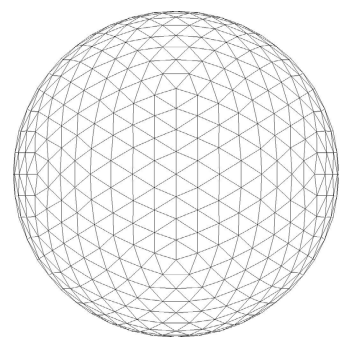

(a)

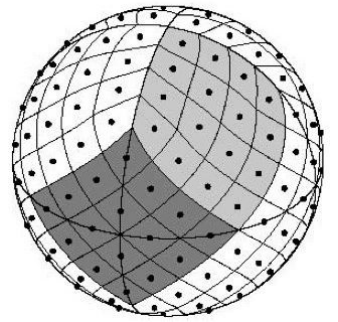

(b)

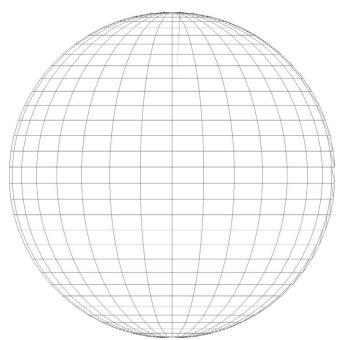

(c)

Fig. 1. (a) Subdivision grid [11; (b) HEALPix grid [14]; (c) Equiangular grid [15]

$L^{1}$ normalized spherical wavelet function $\Psi^{(1)}$ is such as (see proposition 2.1 in [15] 1

$$
\Psi(\theta, \phi)=\Psi^{(1)}(\theta, \phi)=\frac{\Psi^{(2)}(\theta, \phi)}{1+\cos \theta}
$$

\section{Compound Poisson Cascades (CPC) on the Sphere}

A tunable self similar process generated on the sphere is used to validate the multifractal analysis described above. We have chosen the CPC model, a family of multifractal processes that can be defined in $\mathrm{N}$ dimensions [7] and in particular directly on the sphere (without any texture mapping), as explained below.

\subsection{Compound Poisson Cascades in the Plane}

A $2 \mathrm{D} \mathrm{CPC}$ is a stochastic process used to synthesize gray level images. The pixel located at $(x, y)$ takes the value given by

$$
Q_{\ell}(x, y)=\frac{\prod_{\left(x_{i}, y_{i}, r_{i}\right) \in C_{\ell}(x, y)} W_{i}}{\mathbb{E}\left[\prod_{\left(x_{i}, y_{i}, r_{i}\right) \in C_{\ell}(x, y)} W_{i}\right]} .
$$

where $\left(x_{i}, y_{i}, r_{i}\right)$ result from a Poisson point process in the half space above the image plane. The coordinates $x_{i}$ and $y_{i}$ are uniformly distributed on the plane and the $r_{i}\left(\ell \leq r_{i} \leq 1\right)$ are distributed with density $\propto 1 / r_{i}^{3}$. The 3D points are weighted by i.i.d. random multipliers $W_{i}>0$. The pixel value is computed as the product of the multipliers belonging to a cone pointing to this pixel (see Fig. $2(\mathrm{a})$. In another interpretation of (7), the multiplier $W_{i}$ can be seen as a light source influencing a part of the image. The size of its base is dilated depending on the height $r_{i}$ of the multiplier (see Fig. 2(b)]. Such models generate purely multifractal textures characterized by the non linear function $\tau(q)$. For instance, $S_{q}^{b o x}(a) \propto a^{\tau(q)}$. The distribution of the multipliers $W_{i}$ prescribes $\tau(q)$ since

\footnotetext{
${ }^{1}$ We have implemented the $L^{1}$ spherical Mexican Hat wavelet in our YAWTb version.
} 


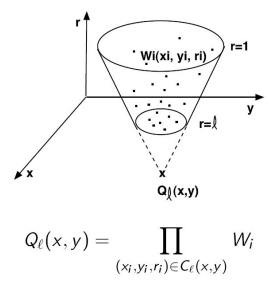

(a)

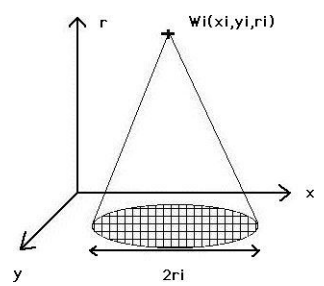

(b)

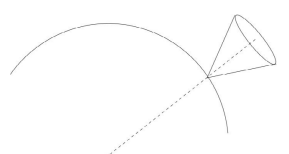

(c)

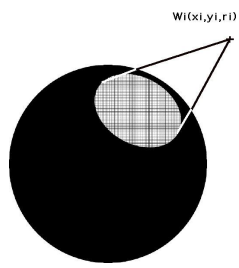

(d)

Fig. 2. IDC construction (a) and (b) in 2D; (c) and (d) on the sphere

$\tau(q)=q\left(\mathbb{E} W_{i}-1\right)+1-\mathbb{E} W^{q}$. These very singular textures are often called bare textures $(H=0)$. More regular and smoother multifractal textures are obtained using a self-similar $1 /\|k\|^{H}(H>0)$ low-pass filter in the Fourier domain. The resulting texture is then characterized by the function $\zeta(q)=q H+\tau(q)$. Such filtered CPC are called dressed textures.

\subsection{Extension of the Compound Poisson Cascades to the Sphere}

The extension of CPC on the sphere uses the same principle as the 2D construction (see Fig. 2(c) and 2(d) . However, several choices are possible to define a dilation on the sphere, e.g. the stereographic dilation or the dilation of a solid angle. We have chosen this last dilation because it is the most direct equivalent of the dilation in the plane and it receives intuitive physical interpretation. The spherical texture (see Fig. 3(a) is then generated by using (7), replacing the $2 \mathrm{D}$ coordinates by spherical ones $(\theta, \phi)$ and adapting the distribution of scales $r_{i}$ (not detailed here for briefness). As explained in Sect. 3.1 the resulting bare spherical texture, characterized by the function $\tau(q)$, has to be filtered to get a dressed texture characterized by $\zeta(q)=q H+\tau(q)$. This filtering is carried out in the (discrete) spherical harmonic domain [17] which is equivalent to the (discrete) Fourier domain. In brief, a function on the sphere $f(\theta, \phi)$ can be expanded on the set of spherical harmonics $Y_{l}^{m}(\theta, \phi)$ of degree $l$ and order $m$

$$
f(\theta, \phi)=\sum_{l \geq 0} \sum_{|m| \leq l} \hat{f}(l, m) Y_{l}^{m}(\theta, \phi) .
$$

where the $\hat{f}(l, m)$ are the spherical harmonic coefficients of $f$. A convolution theorem exists on the sphere to describe azimuthally symmetric filters $\hat{h}(l, 0)$ :

$$
\widehat{(f * h)}(l, m)=2 \pi \sqrt{\frac{4 \pi}{2 l+1}} \hat{f}(l, m) \hat{h}(l, 0) .
$$

Using the rough correspondence between the spherical indices $(l, m)$ and the Fourier vector $\sqrt{l^{2}+m^{2}} \sim k$ [18], we have chosen 


$$
\hat{h}(l, m)=\frac{1}{2 \pi} \sqrt{\frac{2 l+1}{4 \pi}} \frac{1}{{\sqrt{l^{2}+m^{2}}}^{H}} .
$$

as the spherical equivalence of the $1 /\|k\|^{H}$ filter. We know that this filtering is still approximative due to aliasing problems but it appears to be sufficiently precise since for this specific choice, aliasing effects are small. The resulting dressed texture can be characterized by a set of multifractal exponents $\zeta(q)=$ $\tau(q)+q H$ (see Fig. 3(b) .

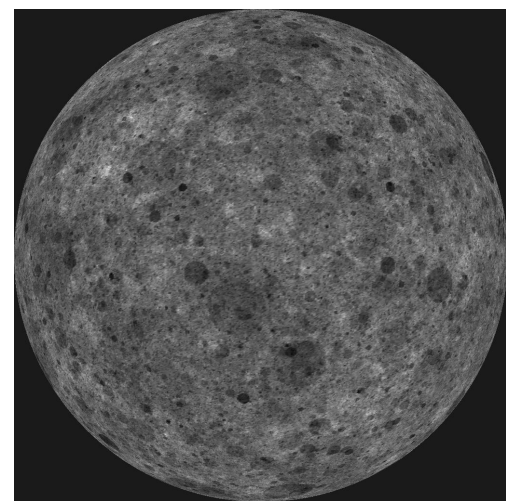

(a)

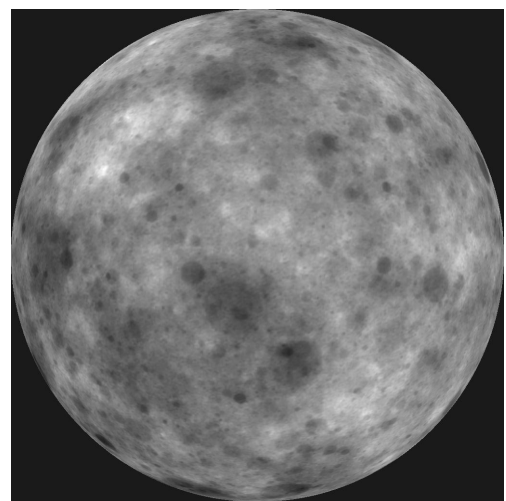

(b)

Fig. 3. CPC textures on the sphere: (a) bare texture characterized by $\tau(q)$ and (b) dressed texture characterized by $\zeta(q)=\tau(q)+q H$

\section{Numerical Study}

The multifractal analysis introduced in Sect.2 is applied on a set of spherical textures generated with the model presented in Sect. 3. The spherical multifractal analysis will give an estimate $\hat{\tau}(q)$ of the exponents $\tau(q)$ prescribed in the synthesis of the CPC processes (see [7] for details). To evaluate the quality of the estimated $\hat{\tau}(q)$, we compare our results to the theoretical $\tau(q)$, to the $\hat{\tau}_{b o x}(q)$ using (11) for bare textures only and to the well-known results of the same experiments performed in the plane. The filtering using spherical harmonics is performed with the S2Kit (http://www.cs.dartmouth.edu/ geelong/sphere/) package [19] and the spherical wavelet transform is done with YAWTb. The wavelet used is the spherical Mexican Hat Wavelet, often picked up in astronomical applications of spherical wavelets [20].

Two sets of 30 spherical and 2D textures corresponding to the so-called logexponential CPC with theoretical $\tau(q)=1-(1+T)^{q} /(1+q T)$ have been computed: bare textures with parameter $T=0.7$ and the corresponding dressed textures with filter parameter $H=0.55$. The choice $T=0.7$ corresponds to very multifractal textures, that is very non linear $\tau(q)$. 
As a first consistency check, we have estimated the multifractal coefficients using the aggregation coefficients method. This analysis can be performed in the direct space. This is quite difficult to implement because of the need to locate the grid vertices and the huge number of iterations. This implementation is also very expensive in computation time and is dependent on the spherical grid. Another implementation trades on the process used in YAWTb to compute wavelet coefficients. This method uses the spherical harmonic transform. It permits an efficient use of the aggregation coefficients mentioned in (1). The results of the aggregation coefficients analysis are presented in Fig. 4. The exponents $\hat{\tau}_{b o x}(q)$ accurately estimate the theoretical $\tau(q)$ which validates the spherical CPC synthesis procedure.

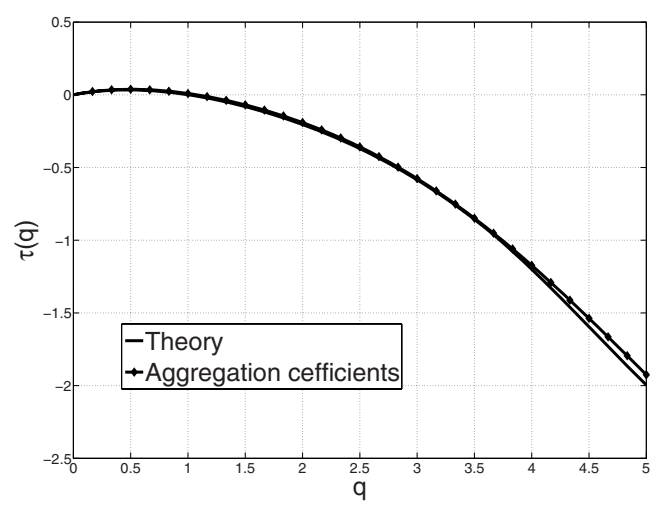

Fig. 4. Estimates based on aggregation coefficients $\hat{\tau}_{b o x}(q)$ for bare textures

Scaling exponents estimates are obtained from linear regressions in $\log _{2} S_{q}(j)$ vs. $j=\log _{2}(a)$ diagrams over 5 octaves. The values of $q$ are chosen in the range $0 \leq q \leq 5$. This range is restricted to positive values because the estimation for negative values is numerically unstable. Furthermore, estimates for $q \geq 5$ are expected to be either statistically inaccurate or uninformative.

Figure 5(a) presents the wavelet-based estimates $\hat{\tau}_{s w t}(q)$ for the bare spherical textures compared to the theoretical values $\tau(q)$ and the results of the same experiments with flat 2D images. The corresponding spherical $S_{q}^{s w t}$ functions present a quite nice power law behavior onto a large range of scales and the behavior (trend and curvature) of $\hat{\tau}_{s w t}(q)$ is quite similar to the theoretical $\tau(q)$. However, SWT-based estimates suffer from systematic bias in contrast with estimates based on box averages $\hat{\tau}_{b o x}(q)$ that were close to perfect. A noticeable defect of $\hat{\tau}_{s w t}(q)$ is that it is not consistent with the fundamental property of bare CPCs $\hat{\tau}_{\text {swt }}(1)=-0.05 \neq 0$. The $2 \mathrm{D}$ equivalent wavelet analysis performed with planar 2D textures based on the same CPC model would give more consistent results.

The behavior (trend and curvature) of $\hat{\zeta}_{s w t}(q)$ is quite similar to the theoretical $\zeta(q)$, see Fig. 5(b). However, we observe again that the $\hat{\zeta}_{s w t}(q)$ systematically underestimate the expected $\zeta(q)$. As a consequence, the parameter $H$ is badly 
estimated since $\hat{H}=\hat{\zeta}_{\text {swt }}(1)=0.46<H=0.55$. This bias is consistent with the bias previously observed on the $\hat{\tau}_{s w t}(q)$ for bare textures. This indicates that the spherical $1 /\|k\|^{H}$ filtering operation seems to work satisfactorily.

For some given model and using equivalent statistics, the variance of spherical estimates is of the same order of magnitude as the variance of $2 \mathrm{D}$ estimates (of the order of $5 \%$, not represented here for sake of clarity).

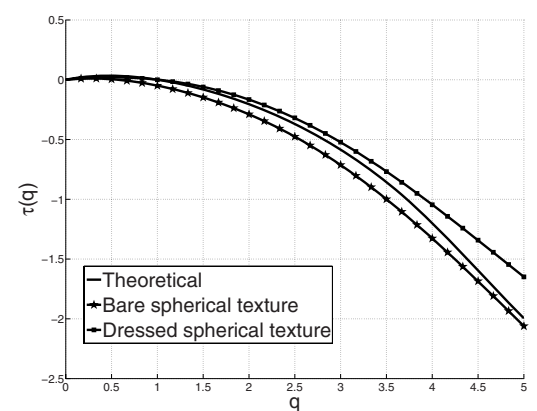

(a)

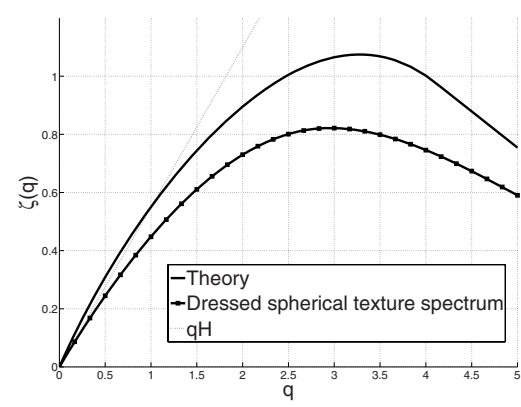

(b)

Fig. 5. (a) SWT $\hat{\tau}_{s w t}(q)$; (b) Multifractal analysis of dressed textures, $\zeta(q)$ of the form $q H+\tau(q)$

\section{Conclusions and Perspectives}

The purpose of this work was to study the relevance of a natural generalization of the wavelet based multifractal analysis from the usual 2D cartesian space to the sphere. Such a tool would be useful to characterize the scale invariance of spherical data. We have presented a numerical study of this approach based on the use of the CPC stochastic processes with prescribed multiscaling properties. These processes can be synthesized directly on the sphere without any mapping artifact. For better efficiency, we have implemented the multifractal analysis based on aggregation coefficients using the YAWTb wavelet transform process. This analysis confirms that the synthetic processes have the prescribed properties indeed. Despite their ability to capture the main behavior of the scaling exponents, the wavelet based estimates $\hat{\tau}_{s w t}(q)$ and $\hat{\zeta}_{s w t}(q)$ are not as accurate as their usual 2D equivalent. Note that many intricate problems arise from the spherical geometry. Indeed, we can not exclude that the chosen mesh grid (equiangular, subdivision, HEALPix,...) have some influence. In particular, theequiangular grid is not a multiresolution grid. This problem is combined to the use of continuous spherical wavelets which doesn't form the desired multiresolution basis commonly used in 2D. This preliminary work shows that the extension of multifractal analysis on the sphere using spherical wavelets may work but is not so immediate. Forthcoming work is needed to get more accurate estimates. 


\section{References}

1. Frisch, U.: Turbulence, The legacy of A. Kolmogorov. Cambridge University Press, Cambridge (1995)

2. Abry, P., Flandrin, P., Taqqu, M., Veitch, D.: Wavelets for the analysis, estimation and synthesis of scaling data. In: Self-Similar Network Traffic and Performance Evaluation, Wiley-Interscience, Chichester (2000)

3. Arneodo, A., Bacry, E., Graves, P.V., Muzy, J.F.: Characterizing long-range correlations in dna sequences from wavelet analysis. Physical Review Letters 74(16), 3293-3296 (1995)

4. Turiel, A., Perez-Vincente, C.J., Grazzini, J.: Numerical methods for the estimation of multifractal singularity spectra on sampled data: a comparative study. Journal of Computational Physics 216(1), 362-390 (2006)

5. Marinucci, D., Pietrobon, D., Balbi, A., Baldi, P., Cabella, P., Kerkyacharian, G., Natoli, P., Picard, D., Vottorio, N.: Spherical needlets for cosmic microwave background data analysis. Mon. Not. R. Astron. Soc. 383(2), 539-545 (2007)

6. Tosic, I., Bogdanova, I., Frossard, P., Vanderghynst, P.: Multiresolution motion estimation for omnidirectional images. In: EUSIPCO (2005)

7. Chainais, P.: Infinitely divisible cascades to model the statistics of natural images. IEEE Trans. on Pattern Anal. Mach. Intell. 29(1) (2007)

8. Mandelbrot, B.: The fractal geometry of Nature. W.H. Freeman and Co, New York (1982)

9. Lashermes, B., Abry, P., Chainais, P.: New insights on the estimation of scaling exponents. Int. J. of Wavelets, Multiresolution and Information Processing 2, 497$523(2004)$

10. Jaffard, S.: Multifractal formalism for functions. S.I.A.M. 28(4), 944-998 (1997)

11. Schröder, P., Sweldens, W.: Spherical wavelets: Efficiently representing functions on a sphere. In: Computer Graphics Proceedings SIGGRAPH 1995, vol. 29, pp. 161-172 (1995)

12. Gao, Y., Nain, D., LeFaucheur, X., Tannenbaum, A.: Spherical wavelet itk filter. In: Ayache, N., Ourselin, S., Maeder, A. (eds.) MICCAI 2007, Part I. LNCS, vol. 4791, Springer, Heidelberg (2007)

13. Starck, J.L., Moudden, Y., Abrial, P., Nguyen, M.: Wavelets, ridgelets and curvelets on the sphere. Astron. astrophys. 446, 1191-1204 (2006)

14. Gorski, K., Hivon, E., Banday, A., Wandelt, B., Hansen, F., Reinecke, M., Bartelmann, M.: Healpix: A framework for high-resolution discretization and fast analysis of data distributed on the sphere. Astrophys. J. 622(2), 759-771 (2005)

15. Antoine, J.P., Demanet, D., Jacques, L., Vandergheynst, P.: Wavelets on the sphere: implementation and approximations. Appl. Comp. Harmon. Anal. 13, 177 $200(2002)$

16. Jacques, L.: Ondelettes, repères et couronne solaire. $\mathrm{PhD}$ thesis, Université catholique de Louvain (2004)

17. Driscoll, J., Healy, J.: Computing fourier transforms and convolutions on the 2sphere. Adv. in Appl. Math. 15, 202-250 (1994)

18. Zhou, K., Bao, H., Shi, J.: 3d surface filtering using spherical harmonics. ComputerAided Design 36(4), 363-375 (2004)

19. Kostelec, P., Rockmore, D.: S2Kit: A Lite Version of SpharmonicKit, Department of Mathematics, Dartmouth College, Hanover (2004)

20. Gonzalez-Nuevo, J., Argueso, F., Lopez-Caniego, M., Toffolatti, L., Sanz, J., Vielva, P., Herranz, D.: The mexican wavelet family: Application to point source detection in cmb maps. Mon. Not. R. Astron. Soc. 369(4), 1603-1610 (2006) 